\title{
CLA Isomer t10, c12 Induce Oxidation and Apoptosis in 3t3 Adipocyte Cells in a Similar Effect as Omega-3 Linolenic Acid and DHA
}

\author{
Meadus WJ*, Vahmani P, Duff P, Zantinge JL, Turner TD and Dugan \\ MER* \\ Agriculture \& Agri-Food Canada, Lacombe, Alberta, Canada
}

\section{Research Article \\ Volume 1 Issue 3}

Received Date: November 07, 2016

Published Date: November 17, 2016

*Corresponding authors: Meadus WJ and Dugan MER, Agriculture \& Agri-Food

Canada, Lacombe, Alberta Canada, Email: Jon.meadus@agr.gc.ca; mike.dugan@agr.gc.ca

\section{Abstract}

Background: Commercial conjugated linoleic acid (CLA) dietary supplements of contain an equal mixture of the C18:2 isomers, cis-9, trans-11 and trans-10, cis-12. CLA-c9t11 occurs naturally in meat and dairy products as the dominant CLA at 75\%, whereas the CLAt10c12 occurs at less than 1\%. CLA-c9t11 generally promotes lipid accumulation but CLA-t10c12 inhibits lipid accumulation and may promote inflammation. The omega-3 fatty acid $\alpha$-linolenic acid (C18:3n-3) and docosahexaenoic acid (DHA) were also observed to inhibit growth of $3 \mathrm{t} 3$ adipose cells; therefore we examined the effects of the CLA vs. omega -3 fatty acids at the molecular level in vitro to determine if they are causing similar oxidative stresses.

Methods: Purified CLA-c9t11 and CLA-t10c12 were added to 3T3 mature adipocyte cultures at 100uM concentrations and compared with 100uM C18:3(n-3) ( $\alpha$-linolenic acid) and 50uM docosahexaenoic acid (DHA) to study their effect on growth, gene transcription and general oxidation. The results of multiple separate trials were averaged and compared for significance at levels of $\mathrm{p}<0.05$, using one way ANOVA and Student's t-test.

Results: C18:3(n-3), DHA and CLA-t10c12 were inhibitory to 3t3 adipose cell growth and caused significant lipid hydro peroxide activity. CLA-t10c12 and c9t11 increased AFABP, FAS and ACOX1 mRNA expression but DHA and C18:3(n-3) decreased the same mRNAs. CLA-c9t11 but not the t10c12 stimulated adipoQ expression even though; c9t11 had only a slightly greater affinity for PPAR $\gamma$ than CLA- t10c12. The expression of the xenobiotic metabolism genes, aldo-keto reductase 1c1 (akr1c1), superoxide dismutase (SOD) and inflammation chemokine secretions of eotaxin (CCL11), Rantes (CCL5), MIG (CCL9) and MCP-1 were increased by DHA, C18:3(n-3) and CLA-t10c12. This correlated with an increase in apoptosis factors, caspase 3, Bcl-2 and BAXs. Apoptosis factors were partially reduced by co-treatment with lipophilic anti-oxidant $\alpha$-tocopherol.

Conclusion: Based on this evidence, CLA-t10c12 promoted more reactive oxygen species (ROS) than CLA-c9t11, in a similar effect as omega 3 fatty acids, C18:3(n-3) and DHA. In response, cascades of genes are activated to deal with the potentially damaging effects of ROS through detoxification, inflammation or apoptosis.

Keywords: CLA-t10c12; CLA-c9t11; Gene expression; Adipocyte lipid hydroperoxide; DHA; 3t3 adipocytes; Apoptosis 


\section{Open Access Journal of Agricultural Research}

Abbreviations: CLA: Conjugated Linoleic Acids; PPAR: Peroxisome Proliferator Activated Receptor; MAPK: Mitogen-Activated Protein Kinase; ACC: Acetyl CoA Carboxylase; FAS: Fatty Acid Synthase; SCD1: Stearoyl CoA delta-9 Desaturase; TNF $\alpha$ : Tumour Necrosis Factor Alpha; COX-2: Cyclooxygenase-2 Gene; ROS: Reactive Oxygen Species; EPA: Eicosapentaenoic Acid; DHA: Docosahexaenoic Acid; FCS: Fetal Calf Serum; BSA: Bovine Serum Albumin; TR-FRET: Time-Resolved Fluorescence Resonance Energy Transfer

\section{Introduction}

Conjugated linoleic acids (CLA) are a group of over 28 naturally occurring C18:2 linoleic acid isomers, containing a conjugated, instead of a methylene, interrupted double bond system. They are found naturally in meat and dairy products derived mainly from plant oil converted by ruminant bacteria [1]. The main natural isomer of CLA is c9, t11-18:2 (rumenic acid), accounting for over $75 \%$ of total CLAs in dairy or meat products [2]. The next most common isomer in milk is CLA-t10c12 but levels typically only reach $\sim 0.1 \%$ in milk $(\sim 10 \mathrm{mg} / \mathrm{kg}$ of fat $)$. CLAt10c12 can be increased to $60 \mathrm{mg} / 100 \mathrm{~g}$ of fat in dairy cattle by feeding a high concentrate low forage diet, which also induces milk depression [3]. The principle dietary source of CLA-t10c12 is from commercial preparations of CLA that were chemically synthesized supplements containing equal mixtures of CLA-c9t11 and -t10,c12 isomers and are marketed (Trofocell@ Hamburg, Germany) (Tonalin $\AA$, Hovdebygda, Norway) with the added health benefits of causing fat oxidation [4]. Feeding a synthetic mixture of 1:1 CLA-c9t11 and CLA-t10,c12 to swine has been shown to reduce fat accumulation and increase lean muscle mass [5,6]. Evidence now shows that the two isomers of CLA, -c9t11 and -t10c12, may have different cellular and metabolic effects.

The effects of dietary CLAs were thought to act mainly through the peroxisome proliferator activated receptor (PPAR) family of transcription factors. Biological activity of CLA-c9t11 is associated with reducing fat, improving insulin sensitivity and possible have anti-carcinogenic effects [7]. CLA-t10c12 treatment is associated with substantial lipid oxidation and peroxidation which may induce by the inflammatory NF-kB and mitogen-activated protein kinase (MAPK) signalling factors $[8,9]$. In mouse liver [10] and human adipocytes, CLA-c9t11 binds with peroxisome proliferator activated receptor (PPAR) which increases fat metabolic enzymes including, acetyl CoA carboxylase (ACC), fatty acid synthase (FAS) and stearoyl coA delta-9 desaturase (SCD1). CLA-t10c12 is also thought to bind with PPAR but prolonged treatment reduces fat metabolic enzymes and increased inflammation markers, tumour necrosis factor alpha $(\mathrm{TNF} \alpha)$ and cyclooxygenase-2 gene (COX-2) [11]. This divergent effect of the two major CLA isomers could be due to effects of their actions as ligands for the PPARs nuclear receptors or to their respective metabolic products acting downstream to influence expression.

Based on this evidence, CLA-t10c12 may promote more reactive oxygen species (ROS) than CLA-c9t11. In response to ROS, a cascade of genes may be activated to either deal with detoxification to prevent potentially damaging effects of ROS or to call out for assistance by the inflammasome [12]. Too much inflammation will also result in damage, leading to apoptosis activation, necrosis and cell death [13]. Similar mechanism dealing with oxidative stress has been speculated to occur with the omega-3 fatty acid, C18:3 ( $\alpha$-linolenic acid) and to a much greater effect by the long chain omega- 3 fatty acids C20:5(n-3) eicosapentaenoic acid (EPA) and C22:6(n-3) docosahexaenoic acid (DHA) $[14,15]$. The oxidative effects of DHA are partially attenuated by co-treatment with the anti-oxidative vitamin E ( $\alpha$-tocopherol) [16]. The objective of the present study was to investigate the high dose effects of CLA-c9t11 and CLA-t10c12 and compare them with omega-3 fatty acids, C18:3(n-3), DHA and DHA with vitamin $E$ treatments, on the stress response in $3 \mathrm{t} 3$ mature adipocytes.

\section{Materials \& Methods}

\section{Cell treatment in culture}

Mouse derived 3T3-L1 cells (ATCC CR-173, Manassas, VA, USA) were used to characterise the effects of the free fatty acids. The 3T3-L1 cells in their pre-adipocyte stage were grown in Dulbecco modified eagle's media (DMEM) $+10 \%$ fetal calf serum (FCS) + $10 \mathrm{U} / \mathrm{ml}$ penicillin/streptomycin (Thermo Fisher Life Technologies, Burlington, ON, Canada). Induction of 3t3 cells to mature adipocytes began after the cultures reached $>90 \%$ confluence. 3 T3 cells were induced into terminally differentiated mature adipocyte by treatment with $10 \mu \mathrm{g} / \mathrm{ml}$ insulin $+0.1 \mu \mathrm{M}$ 3-isobutyl-1methylxanthine (IBMX) and $0.5 \mathrm{mM}$ dexamethasone in DMEM (Life technologies, Burlington, ON Canada) $+10 \%$ fetal bovine serum for $48 \mathrm{~h}$ and then prolonged incubation in by DMEM + 10\% FBS + $10 \mu \mathrm{g} / \mathrm{ml}$ insulin (cat\# I9278, Sigma-Aldrich Canada, Oakville, ON, Canada) . Mature adipocyte cultures $(n=6)$ were visually assessed by the appearance of lipid droplets in the cells which became apparent under 10X magnification after 4 to $7 \mathrm{~d}$ in culture 


\section{Open Access Journal of Agricultural Research}

(Figure 1). Treatments of the 3t3 mature adipocytes with the free fatty acids were complexed with $7.5 \%$ fatty acid free bovine serum albumin (BSA) at $4 \mathrm{mM}$ to insure solubility. All fatty acid solutions were initially solubilized in $100 \%$ dimethyl sulfoxide (DMSO) then diluted to the appropriate concentrations in growth media. CLA-c9t11 (9Z,11E-octadeca-9,11-dienoic acid) , CLA-t10c12 (10E,12Z-octadeca-9,11-dienoic acid), C18:3(n-3) (all-cis9,12,15-octadecatrienoic acid), C22:6n-3 (all-cis-docosa4,7,10,13,16,19-hexa-enoic acid, DHA) and linoleic acid (18:2, all-cis-9,12-octadecadienoic acid) were purchased from Nu-Chek Prep Inc. (Elysian, MN, USA). DL-all-racAlpha-tocopherol acetate was purchased from Sigma cat\# T3251 (Sigma Aldrich Canada, Oakville, ON, Canada).
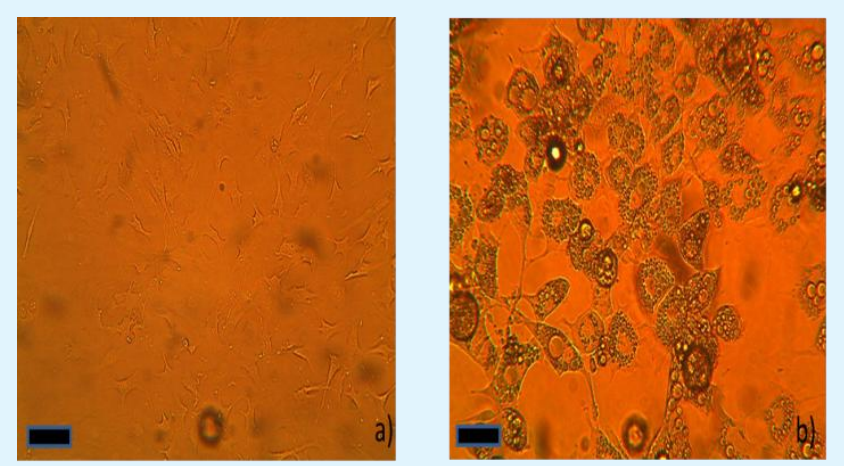

Figure 1: Phase microscope 10X image of $3 \mathrm{t} 3$ cells.

a) pre-adipocyte form and as b) mature adipocytes after 7 days treatment and incubation in culture media DMEM + 10\% fbs with $10 \mathrm{ug} / \mathrm{ml}$ insulin. Note the appearance of lipid droplets in the mature adipocyte cells. Scale bar equals $100 \mu \mathrm{m}$.

\section{Assessment of cell viability with Thiazolyl Blue Tetrazolium Bromide (TBTB/MTT)}

Cell viability was assessed using 3-4,5-Dimethylthaizol2-yl)-2,5-diphenyl tetrazolium bromide assay [17]. Essentially, the cells were incubated with $10 \mathrm{mg} / \mathrm{ml}$ of TBTB/MTT in the growth media for 4hours. The metabolic activity of cells was measured by the conversion of yellow TBTB/MTT into blue MTT-formazan by the mitochondrial dehydrogenase [18]. The growth media was removed, rinse the adherent cells with phosphate buffered salt solution and develop the colour with $100 \%$ DMSO for $12 \mathrm{hr}$ and read the absorbance at $570 \mathrm{~nm}$.

\section{In vitro competitive binding studies with PPARg}

Competitive binding assays were performed in vitro using LanthaScreen time-resolved fluorescence resonance energy transfer (TR-FRET) method (Invitrogen, Carlsbad,
CA). The fluormone Pan-PPAR green ligand competed with unlabelled CLA-c9t11, CLA-t10,c12, linoleic acid (LA 18:2-cis9, cis12) and GW1929 (To Cris Biosciences, Bistol, UK), which is a known PPARg agonist, for the binding site of a recombinant human PPARg - GST protein which was monitored with a terbium-labelled anti-GST antibody. The half-maximal inhibitory concentration (IC50) value of each test compound was assessed using a sigmoidal dose-response equation (GraphPad Prism 5.0, GraphPad Software, La Jolla, CA, USA).

\section{Gene expression analysis of lipid metabolism, lipid oxidation and apoptosis}

Total RNA was isolated using TRIzol Reagent (Life Science Technologies, ON, Canada) and purified using the Aurum Total RNA kit (Bio-Rad Laboratories, Mississauga, ON, Canada). Total RNA $100 \mathrm{ng} / \mathrm{ml}$ was converted to cDNA using MMLV reverse transcriptase (Bio Basic Inc, Markham, ON Canada) oligo-dT 12-18 at $500 \mathrm{ug} / \mathrm{ml}$ and random hexamers at $125 \mathrm{ug} / \mathrm{ml}$. Gene expression levels of fat metabolic gene included PPAR $\gamma$, fatty acid binding protein 4 (FABP4 also known as $\sim \mathrm{AFABP}, \mathrm{aP} 2$ ), fatty acid synthase (FASN), stearoyl CoA desaturase( SCD1), acyl CoA oxidase(Acox1), carnitine palmitoyl transferase 1A (cpt1a), CIDEA, and adipoQ ( adiponectin, Acrp30) transcripts, were compared using qPCR. $\beta$-actin, RP2A, HPRT and b-microglobulin were used as the reference genes [19]. Gene expression was converted from their cycle threshold (Ct) by $2-\Delta \Delta \mathrm{Ct}$ method [20].

Gene expression of fat oxidation biomarkers were initially determined by microarray analysis using expression microarray panel PAMM-065Z (Qiagen Canada, Mississauga, ON). Total RNA was collected from the CLA-c9t11 and CLA-t10c12 treatments of 100uM for $48 \mathrm{~h}$ and was converted to cDNA with a $\mathrm{RT}^{2}$ First Strand Kit (Qiagen). Using the $\mathrm{RT}^{2}$ SYBR Green polymerase chain reaction (PCR) Master mix according to manufacturer's recommendations on a Mx3000P QPCR real time PCR machine with SYBR gene detection (Agilent Technologies Canada Inc., Mississauga, ON). Quantitative gene analysis was based on comparative $2^{-\Delta \Delta \mathrm{Ct}}$ method [20] using the $\mathrm{RT}^{2}$ profiler PCR data analysis program 3.5. (SA Biosciences/Qiagen, Mississauga, ON). A list of 5 genes identified by the array as being the significantly induced by CLA-t10c12 relative to the CLA-c9t11 treatment were further tested in an oxidation panel by qPCR along with the other cells treatments as previously noted. The expression of mRNA was apoptosis was also tested on the treated cells by qPCR. Primers (presented in Table \#1) were checked for expression according to the minimal information for quantitative real-time PCR experiments ( 


\section{Open Access Journal of Agricultural Research}

MIQE) guidelines. [21]. Normalization of qPCR data [22] was done against the reference genes, $\beta$-actin, RP13A and
HPRT and samples treatments were adjusted against the C18:2 treatments harvested at the same time.

\begin{tabular}{|c|c|c|c|c|}
\hline Genea $^{\mathbf{a}}$ & Forward primer $5^{\prime} \rightarrow 3^{\prime}$ & Reverse Primer $5^{\prime} \rightarrow 3^{\prime}$ & Size (bp) & Source Genbank No. \\
\hline RP13A & ggctgccgaagatggcggag & gccttcacagcgtacgaccacc & 130 & NM_009438 \\
\hline$\beta$-actin & cctctatgccaacacagtgc & cctgcttgctgatccacatc & 185 & NM_007393 \\
\hline HPRT & tgacactggcaaaacaatgca & aggtccttttcaccagcaagct & 94 & NM_013556 \\
\hline SOD3 & atggagctaggacgacgaag & ctcaggtccccgaactcatg & 229 & NM_011435 \\
\hline AKR1c1 & gcggcaggatctcttcattg & gttccatagccgtccaagtg & 232 & D32250 \\
\hline CCL5 & atatggctcggacaccactc & cttcttctctgggttggcac & 163 & NM_013653 \\
\hline MCP-1 & agcaccagccaactctcac & tctggacccattccttcttg & 300 & NM_011333 \\
\hline CASPASE-3 & aaggagcagctttgtgtgtg & ggcaggcctgaatgatgaag & 160 & NM_001284409 \\
\hline Bax & agatcatgaagacaggggcc & gtccacgtcagcaatcatcc & 199 & NM_007527 \\
\hline Bcl-2 & aactcttcagggatggggtg & atcaaacagaggtcgcatgc & 226 & NM_009741 \\
\hline PPARg & ctgtggggataaagcatcag & tgatggcattgtgagacatc & 202 & XM_006505737.1 \\
\hline CPT1 & tcgacaggtggtttgacaag & gaatgtcccactgtagcctg & 221 & NM_013495 \\
\hline AdipoQ & gctcctgctttggtccctccac & gcccttcagctcctgtcattcc & 177 & NM_009605.4 \\
\hline FABP4 & tttctcacctggaagacagc & cttcaccttcctgtcgtctg & 287 & NM_024406 \\
\hline ACOX1 & gataaactccccaagattcaa & aagtcaaaggcatccaccaaa & 214 & NM015729.2 \\
\hline FASN & acacatcctaggcatccgag & tttcctgaagtttccgcagc & 173 & NM_007988.3 \\
\hline CIDEA & tgctcttctgta tcgeccagt & gccgtgttaaggaatctgctg & 112 & NM007702.2 \\
\hline
\end{tabular}

Table 1: Gene specific forward and reverse primer sequences of genes used for QPCR.

aRP13A ribosomal protein large 13A, b-actin beta-actin, HPRT hypoxanthine ribosyltransferase, SOD3 superoxide dismutase 3, ARK1C1 aldo keto reductase isoform 1c1, CCL5 chemokine 5, MCP-1 monocyte chemoattractin protein 1, caspase-3 cystein- aspartic protease 3, BAX apoptosis regulator protein, Bcl-2 b-cell lymphoma 2 apoptosis regulator, PPARg peroxisome proliferator activated receptor gamma, CPT1 carnitine palmitoyl transfer protein 1, AdipoQ adiponectin, FABP4 fatty acid binding protein 4, ACOX1 peroxisomal acyl-coenzyme A oxidase 1, FASN fatty acid synthase, CIDEA cell death activator.

\section{Expression of secreted chemokines}

Cell culture media was adjusted to DMEM with reduced fetal bovine serum at $0.5 \%$ on mature adipocytes for $24 \mathrm{~h}$ or before starting fatty acid treatments to aid in the detection of cytokines. Complete removal of serum severely compromised the $3 \mathrm{t} 3$ mature adipocytes survival. Treatments were started in the BSA supplemented media and the cells or cell media was collected in $100 \mathrm{ul}$ aliquots and kept at $-80 \mathrm{C}$ until analysis. Detection of the chemokines was performed by Eve Technologies Inc. (Calgary, AB, Canada). The bead analyzer (Bio-Plex 200) includes a dual-laser system and a flow-cytometry system. One laser activates the fluorescent dye within the beads which identifies the specific analyte. The second laser excites the fluorescent conjugate (streptavidin-phycoerythrin) that has been bound to the beads during the assay. The amount of the conjugate detected by the analyzer is in direct proportion to the amount of the target analyte. The proteins were quantified using a standard curve. A Bio-Plex 200 machine (Eve Technologies) was used to identify fluorophore signatures for each target cytokine in Mouse 


\section{Open Access Journal of Agricultural Research}

Cytokine Array / Chemokine Array 31-Plex Cat \#: MD31 and adiponectin. Targets in the array included:CCL-5 (RANTES), CCL-11, CxCl-1 (KC), MCP-1 CCL-11 (Eotaxin), G-CSF, GM-CSF, IFN gamma, IL-1alpha, IL-1beta, IL-2, IL-3, IL-4, IL-5, IL-6, IL-7, IL-9, IL-10, IL-12 (p40), IL-12 (p70), IL-13, IL-15, IL-17A, IP-10, KC, LIF, LIX, MCP-1, M-CSF, MIG, MIP-1alpha, MIP-1beta, MIP-2, RANTES, TNF-alpha, VEGF. Protein concentrations were recorded for the serum free media using the bicinchoninic acid (BCA) assay kit (Pierce, Life Technologies Inc., Burlington, ON, Canada).

\section{Lipid Hydroperoxide Assay}

LPO assay kit was obtained from Cayman Chemicals (Ann Arbour, MI, USA). 3t3 mature adipocytes samples were treated in $9 \mathrm{~cm}^{2}$ cultures for $24 \mathrm{~h}, 48 \mathrm{~h}$ and $72 \mathrm{~h}$ and were harvested in protein assay RIPA buffer [25mM Tris$\mathrm{HCl} \mathrm{pH} 7.6,150 \mathrm{mM} \mathrm{NaCl}, 1 \% \mathrm{NP}-40,1 \%$ sodium deoxycholate, $0.1 \%$ SDS] and homogenized on ice. The samples [500ul] were then extracted with 500ul methanol and $1 \mathrm{ml}$ of chloroform. The methanol/chloroform extract [450ul] was then mixed with $0.25 \mathrm{uM}$ ferrous sulfate, $10 \mathrm{mM} \mathrm{HCl}$ and $0.15 \%$ $\mathrm{NH}_{4} \mathrm{SCN}$ and read at $500 \mathrm{~nm}$ to detect iron thiocyanate. The absorbance was compared using a 13-HpODE (13hydroperoxy octadecadanoic acid) standard curve.

\section{Statistical analysis}

The competitive binding TR-FRET PPARg assay was analysed using non-linear regression and graphed using the Prism Graph Pad software. The assay was performed over a concentration range of $0.001 \mathrm{uM}$ to $100 \mathrm{uM}(0.28$ $\mathrm{ng} / \mathrm{ml}$ to $0.28 \mathrm{mg} / \mathrm{ml}$ ) with at least 4 replicates. The means on standard deviations on the MTT ad lipid hydroperoxide assays were compared using Student ttest. The LPO assays were measured using a standard curve based on 13-hydroperoxy octadecadienoic acid. The gene expression measures were compared by analysis of treatment means after adjusting expression levels relative to C18:2 values. Means were compared between groups according to ANOVA and then t-test and Tukey's test using Graph Pad Prism software (La Jolla, CA, USA).

\section{Results}

\section{Cell Viability as determined by MTT assay}

Treatment of the 3T3 mature adipocytes showed a significant inhibitory effect when exposed to $100 \mathrm{uM}$ doses of 18:3, CLA-t10c12. (Figure 2) There was too much inhibition of DHA treated cultures at $100 \mathrm{uM}$ and it was reduced to $5 \mathrm{uM}$, which still showed a significant degree of $66 \%$ inhibition after $72 \mathrm{~h}$. The inhibition of cell activity by 5 uM DHA was reduced by co-culturing with $100 \mathrm{uM}$ alpha-tocopherol to only $51 \%$ of the C18:2 activities but not completely to control levels. The CLA-c9t11 treated cultures appeared to experience some inhibition after $72 \mathrm{~h}$ but this was not significant. In preparation for this experiment, the mature adipocytes experienced too much inhibition according to the MTT activity assay at 250uM treatment after $24 \mathrm{~h}$ to be a useful model of investigation [data not shown].

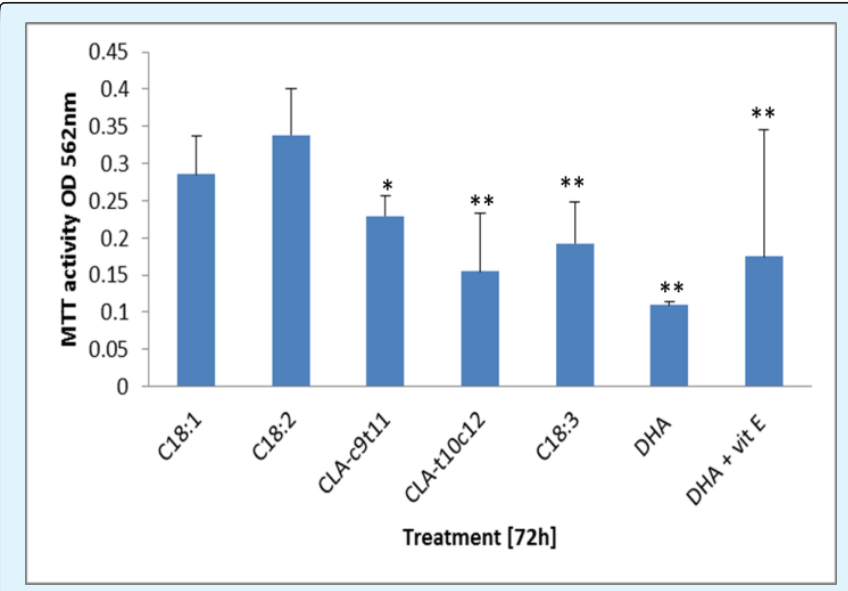

Figure 2: Measure of survival of $3 \mathrm{t} 3$ mature adipocytes by MTT assay.

The free fatty acids were loaded at $100 \mathrm{uM}$ into the media and incubated for $72 \mathrm{~h}$, except $50 \mathrm{uM}$ DHA and $\alpha$-tocopherol (vit E). Survival of cellular activity is represented by the conversion of MTT into blue formazan to give OD reading of $562 \mathrm{~nm}$. Data represent the average from 5 replicates of cell cultures treated. Standard deviations are given as error bars. Significance difference from the C18:2 treated cells is show as $\left({ }^{* *} \mathrm{P}<0.01,{ }^{*} \mathrm{P}<0.05\right.$, \# $\left.\mathrm{P}<0.1\right)$.

\section{Lipid hydroperoxide activity}

The measurement of lipid peroxides in the treated culture was used to indirectly assess the amount of oxidative stress the cells were undergoing at $24 \mathrm{~h} 48 \mathrm{~h}$, and $72 \mathrm{~h}$ treatment. (Figure 3) The assay directly measured the amount of hydroperoxides which are able to react with ferrous ions to produce ferric ions which are then detected by thiocyanate which acts as a chromophore. The results of the assays, adjusted according the protein in the sample, showed that the culture treatments were not significant at $24 \mathrm{~h}$ and were generally unchanged by $48 \mathrm{~h}$. On day 3 (72h) treatment effect of the free fatty acid were quite pronounced with the C18:3(n-3) at 100uM and 


\section{Open Access Journal of Agricultural Research}

DHA at 50uM treated culture experiencing the most hydroperoxides in culture. The CLA-t10c12 also caused a significantly increased by day 3 and this was close to the amount in the C18:3(n-3) treated cells. CLA-c9t11 also had a showed an increase in the lipid hydroperoxides by day 3 but this was not significant relative to C18:2. The co-treatment of DHA with the vitamin E showed that the peroxides were not being generated at the same levels in the $24 \mathrm{~h}$ and $48 \mathrm{~h}$ treatment but this protection was becoming less evident after $72 \mathrm{~h}$ co-treatment with DHA.

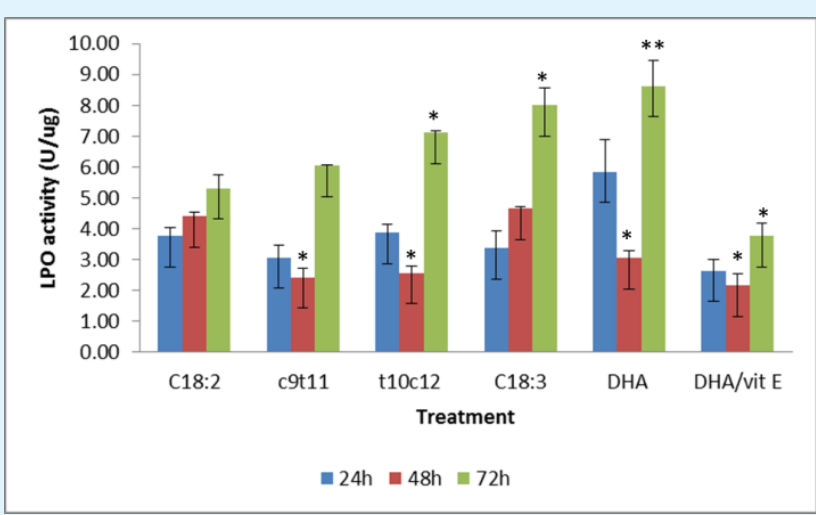

Figure 3: Lipid hydroperoxide activity in mature 3T3 adipocytes treated for $24 \mathrm{~h}, 48 \mathrm{~h}, 72 \mathrm{~h}$ with free fatty acids.

Lipid hydroperoxides were measured by the reduction of ferrous sulfate ions which react with ammonium thiocyanate in chloroform and methanol. Data shown is the cells treated for up to $72 \mathrm{~h}$ with $100 \mathrm{uM}$ of fatty acids except the 50 uM DHA and DHA + vitamin E. Bars represent treatment mean \pm SE. Significant different from the $\mathrm{C} 18: 2$ average. ${ }^{* *} \mathrm{P}<0.01,{ }^{*} \mathrm{P}<0.05$.

\section{Expression of secreted chemokines}

The 31 secreted chemokine array panel showed an increase of RANTES (CCL1) up 60X, MCP-1 up 20X, Exotaxin (CCL11) up 150X, KC (CxCL1) up 20X, VEGF up $4 \mathrm{X}$, and AdipoQ up 1.8X, after the 48h treatment with C18:3(n-3), relative to time 0 (Figure 4). DHA treatment stimulated the VEGF up 4X, CxC1 up 15X and AdipoQ up $1.8 \mathrm{X}$ after $48 \mathrm{~h}$ relative to time 0 . The CCL5, CCL11, CxCl1, MCP-1, VEGF and CCL-5 also known as RANTES are chemokine attractant normally expressed in T-cells and inflammation. It has been reported that these cytokines are activated by long term exposure of $3 \mathrm{t} 3$ mature adipocytes to CLA t10c12 [23] but relative to the C18:2 treatment, only the $\mathrm{CxC} 1$ and $\mathrm{CC} 11$ secreted protein were stimulated after $48 \mathrm{~h}$ (figure 4c,4e). MCP1 (monocyte chemo-attractant protein 1) was stimulated by C18:3(n-3) and DHA relative to C18:2 treatments. CCL11 also known as eosinophil chemotactic protein and $\mathrm{CxCl} 1$ (chemokine (C-X-C motif) ligand 1) are associated with inflammation and their expression were stimulated significantly $(\mathrm{p}<$ 0.05) C18:3(n-3) and CLA-t10c12 insignificantly ( $<<0.1)$ in the $48 \mathrm{~h}$ cultures. The c9t11 treated cultures were not stimulated to secrete cytokines relative to the C18:2 treated cells. Vascular endothelia growth factor (VEGF) is secreted as a chemoattractant and is also expressed in hypoxic conditions and was particularly stimulated by C18:3(n-3) and DHA treatment. Secreted Adiponectin protein did show an increase in the DHA and C18:3(n-3) treated $3 \mathrm{t} 3$ culture media and was significantly stimulated $(\mathrm{p}<0.05)$ according to the $\mathrm{qPCR}$ results (Figure 6). The CLA-c9t11 showed no increase in AdipoQ protein secretion into the media but the qPCR results suggested that the AdipoQ transcript was stimulated by CLA-c9t11 treatment (Figure 6).

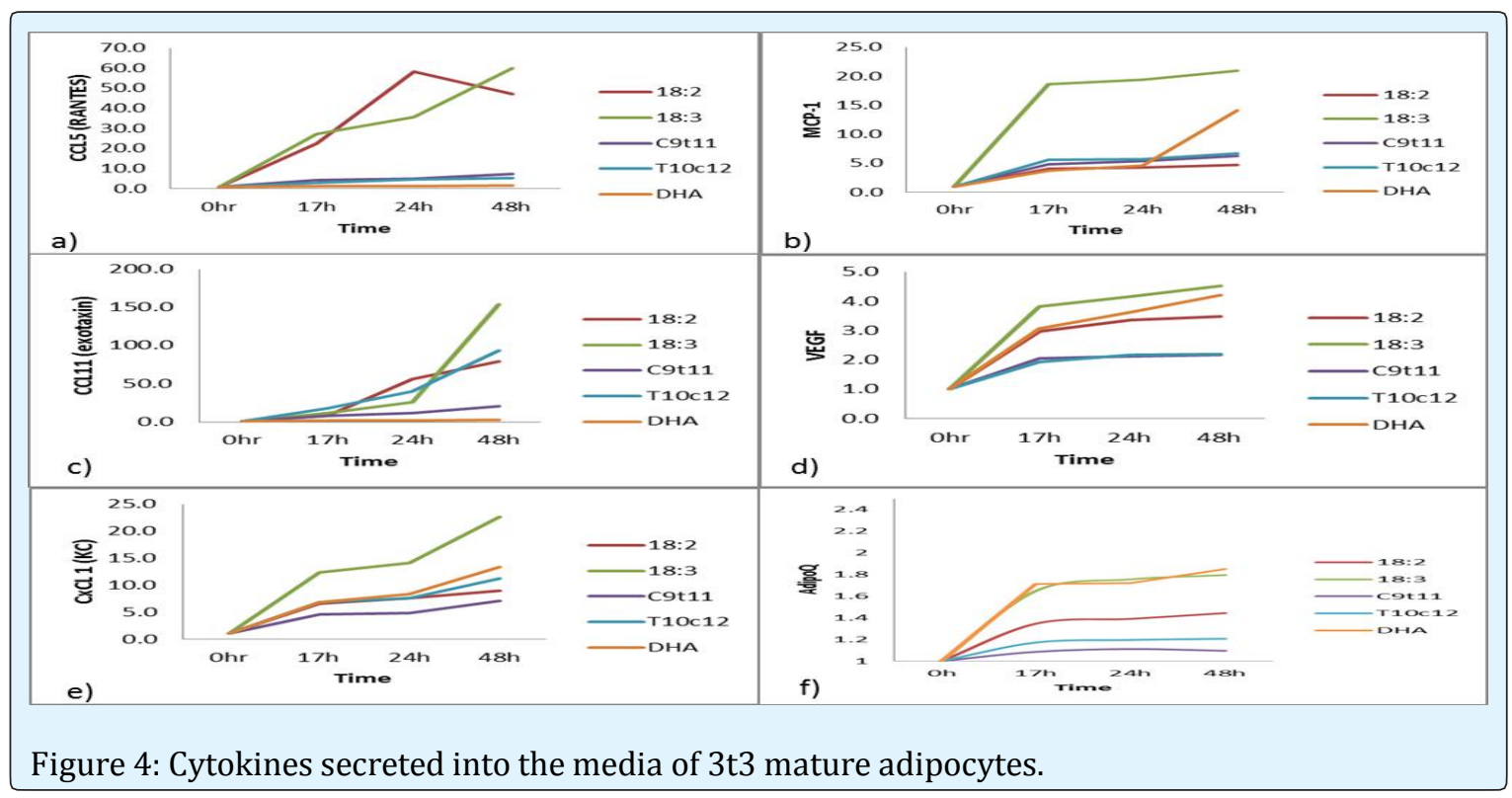




\section{Open Access Journal of Agricultural Research}

The increase of the cytokines CCL5 (RANTES), b) MCP-1, c) CCL11, d) VEGF, e)CxCl1 (KC), f) AdipoQ proteins were measured for 48h in the presence of 100uM C18:2, C18:3, CLA-c9t11, CLA-t10c12, or 50uM DHA, in DMEM media with reduced serum at $0.5 \%$. The data represent the fold increase in the level of the cytokine divided by the estimated level at $\mathrm{t}=0$.

\section{TR-FRET Assay of PPAR $\gamma$ binding}

According to the TR-FRET PPAR-gamma receptor competitive binding assay, the CLA-c9t11 bound at $5.4 \mathrm{uM}$ which was less than either the CLA-t10, c12 at $1.3 \mathrm{uM}$ or the linoleic acid $(18: 2-c 9, c 12)$ with an IC50 of $1.2 \mathrm{uM}$. This was in comparison to the strong synthetic PPAR-gamma agonist GW1929, which bound at IC50 of 0.09 uM. (Figure 5) These values are in agreement with other studies who have looked at the free fatty acids binding PPARg independently but the ability of the PPAR to form complexes with other transcription factors such as retinoic acid receptor (RXR) complicates the in vitro study of ligands such as DHA.

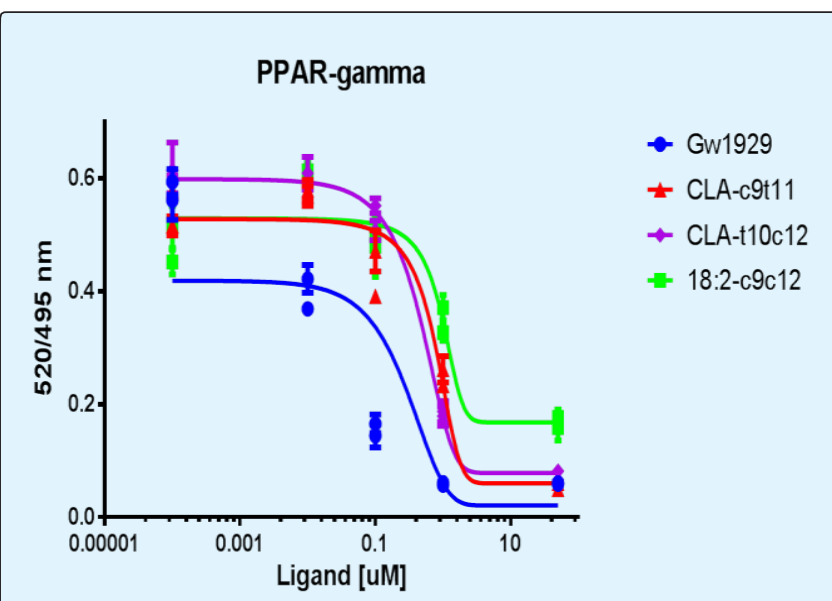

Figure 5: Binding of CLA-t10c12 and CLA-c9t11 to PPAR $\gamma$.

The binding of CLA were measured in a TR-FRET assay to recombinant PPAR $\gamma$. The control substance GW1929 was bound to the PPAR $\gamma$ at competitive IC50 of $0.9 \mathrm{uM}$.

\section{Expression of lipid metabolic markers}

The expression of the fat metabolism genes, ACOX1, FASN, and FABP4 were generally up regulated in the c9t12 treated cells from the C18:2 treated cells and the trend was also seen in the $110 \mathrm{c} 12$ treated cells but the amount was distinctly less $\mathrm{P}<0.05$. (Figure 6) The expression of FAS and ACOX1 was significantly inhibited by C18:3 and DHA treatment but the AdipoQ and FABP4 transcripts were stimulated relative the C18:2. In the DHA and vitamin E treated samples, the AdipoQ, and FABP4 expression was also stimulated but the FASN was inhibited. AdipoQ (aka. adiponectin, Acrp30) is secreted in the blood by adipose tissue and is generally associated an increased sensitivity to insulin and promotes betaoxidation of fats. AdipoQ is also associated increased by dietary EPA and DHA [24]. The transcripts of PPARg, CPT1 and CIDEA a biomarker of lipid droplet size, were all not significantly increased by the high dose treatments but the C18:3(n-3) and DHA suppressed expression of FASN and CIDEA indicating a reduction in new fatty acid accumulation. The most distinguishing factor is that AdipoQ is stimulated by omega-3 fatty acids, C18:3 (n-3) and DHA and not by CLA-t10c12, which agrees with other studies [25].

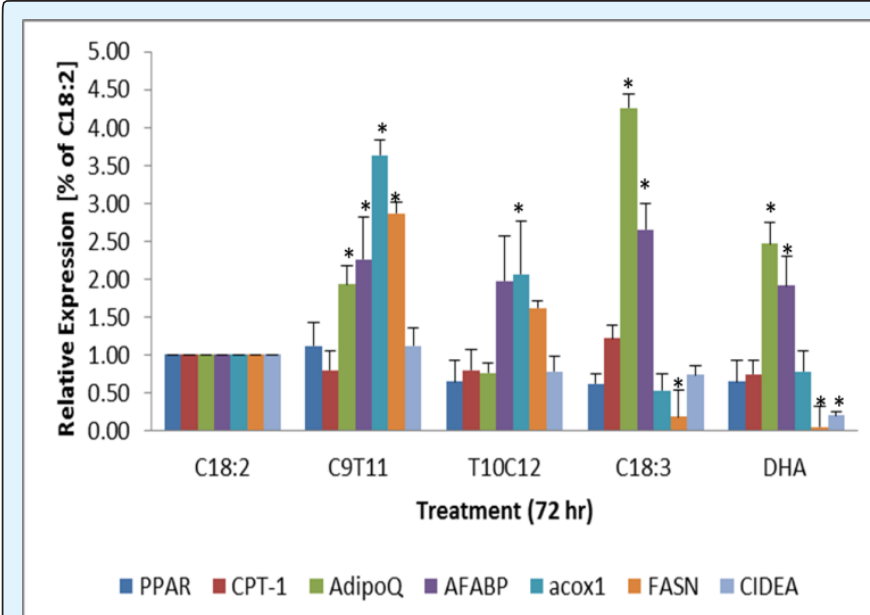

Figure 6: The expressions of lipid metabolism are differentially expressed between CLA and omega- 3 fatty acid treated 3T3 mature adipocytes.

\section{Expression of lipid oxidative factors}

The activity observed in the lipid hydroperoxide activity assay prompted a more in-depth investigation into oxidative enzymes biomarkers. The initial screening of the oxidative biomarkers was first attempted with Qiagen oxidative macroarray PAMM-065Z comparing CLA- c9t11 against CLA-t10c12 72h treated cells. A panel of 90 genes were investigated and suggested that some were differentially expressed more strongly in the CLAt10c12 treated cells. These were the solute carrier protein 38C (SLC38c), duel oxidase (Duox1), cytoglobulin (cygb), superoxidase 3 (SOD3), glutathione peroxidase 3 (Gpx3), and chemokine 5 (CCL5) also known as RANTES [data not shown]. A more detailed investigation into the expression 


\section{Open Access Journal of Agricultural Research}

of these genes and aldo keto reductase 1c1 (ark1c1), which had been noted to be stimulated by lipid oxidation, were performed by qPCR. The expression of ark1c1 was significantly affected by both CLA c9t11 and t10c12 but ark1c1 was more clearly stimulated by $110 \mathrm{c} 12$ treatment after $72 \mathrm{~h}$. (Figure 7) CLA-t10c12 also stimulated the expression of CCL5 and SOD3 genes in the $72 \mathrm{~h}$ treated cells. DHA and C18:3 (n-3) treatments showed a trend (P $<0.05$ ) towards stimulation of CCL5 and SOD3 after 72h. This was reduced by the co-treatment of DHA with $100 \mathrm{uM}$ alpha-tocopherol (vitamin E). MCP-1 was unique as being significantly up regulated by C18:3, DHA and CLA-t10c12 but not by CLA-c9t11. MCP-1 protein secretion into the media was only observed in the omega- 3 treated cultures. The cells were treated for $72 \mathrm{~h}$ with the fatty acids at $100 \mathrm{uM}$ except for the DHA which was treated at 50uM. The transcripts were analysed via qPCR. Means +/standard error $(n=5)$ were compared with C18:2 expressions. Significantly different from the control C18:2 are represented by $* \mathrm{P}<0.05$.

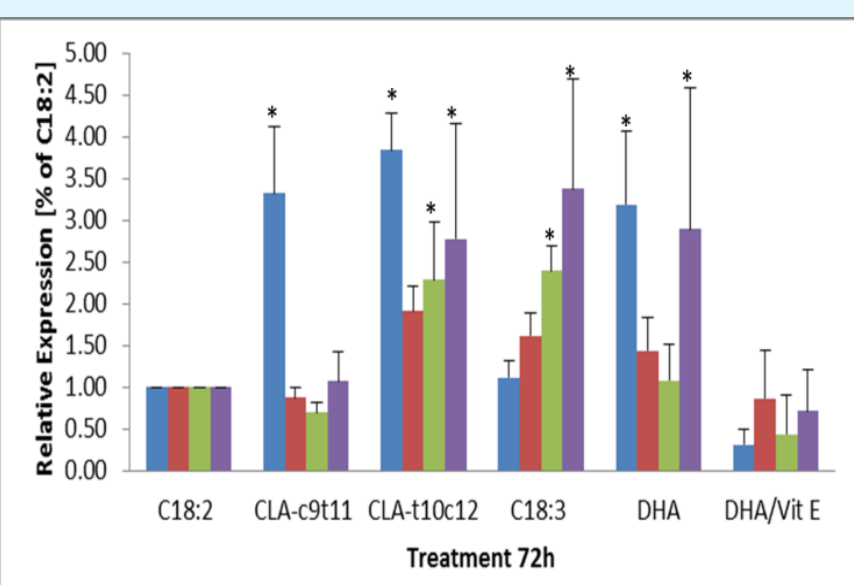

$\approx A R K 1 C 1 \backsim$ SOD3 $\backsim C C L 5 \backsim M C P-1$

Figure 7: The expression of lipid oxidation genes in response to omega-3 or CLA fatty acid treated 3T3 mature adipocytes.

$3 \mathrm{t} 3$ mature adipocytes were treated for $72 \mathrm{~h}$ and the transcripts of the gene ark1c1, SOD3, CCL5 and MCP-1 were evaluated. The transcripts were analysed via qPCR. Means +/- standard error $(n=5)$ were normalized against with C18:2 expressions. Significantly different from C18:2 control are represented by $* \mathrm{P}<0.05$.

\section{Expression of apoptosis biomarkers}

The expression of genes involved in oxidative detoxification and xenobiotics and the accumulation of lipid hydroperoxides suggested that the cells were being stressed and that the environment was inducing lipid toxicity. According to the MTT assays, DHA was tolerated at 50uM in the mature adipocytes and the C18:3(n-3) and CLA-t10c12 at 100uM but still the cultures were showing considerable inhibition, when compared to CLA-c9t11. The expression of caspase $3, \mathrm{Bcl} 2$, and BAX were selected as indicators of cell apoptosis but not senescence. Apoptosis is difficult to properly assess in the fibroblast $3 \mathrm{t} 3$ cell line but after they have undergone terminal differentiation into a mature adipocyte phenotype, the signalling cascade becomes more repeatable. (Figure 8) Apoptosis signals generated from the qPCR measurements were still highly variable. All three of the apoptosis biomarkers, caspase $3, \mathrm{Bcl} 2$, and $\mathrm{BAX}$ were up significantly in the culture treated with 50uM DHA at $24 \mathrm{~h}$ but after $72 \mathrm{~h}$, the BAX signal showed a significant decline relative to the C18:2. C18:3(n-3) showed an 3x- 6x (P< 0.05 ) increase in caspase 3 by $48 \mathrm{~h}$ and $72 \mathrm{~h}$ treatment and a $4 \mathrm{x}$ increase in the $48 \mathrm{~h} \mathrm{Bcl} 2$ signal. CLA-t10c12 treatment significantly increased the caspase 3 in the $72 \mathrm{~h}$ treatment, the BAX signal in the $24 \mathrm{~h}$ and $48 \mathrm{~h}$ samples and just the $48 \mathrm{~h}$ Bcl2 signal. The CLA-c9t11 only increased the 24h BCl-2 expression signal slightly and not the caspase 3 or BAX signals.

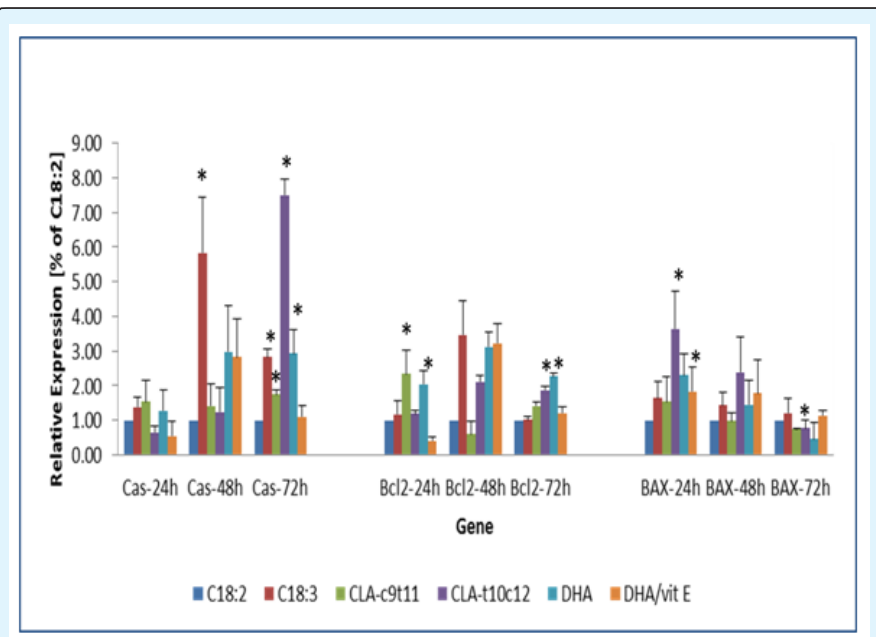

Figure 8: The expression of apoptosis biomarker genes in response to omega-3 or CLA fatty acid treated 3T3 mature adipocytes.

$3 \mathrm{t} 3$ mature adipocytes were treated for $72 \mathrm{~h}$ and the transcripts of the genes caspase $3, \mathrm{Bcl}-2$ and BAX were evaluated. The transcripts were analysed via qPCR. Means +/- standard error $(n=3)$ were normalized against with C18:2 expressions. Significantly different from the control C18:2 are represented by, ${ }^{* *} \mathrm{P}<0.01,{ }^{*} \mathrm{P}<0.05$. 


\section{Open Access Journal of Agricultural Research}

\section{Discussion}

The subtle differences between CLA-c9t11 and CLAt10c12 were compared to omega 3 fatty acids C18:3 and DHA to examine if they caused similar stress mechanism in mature $3 \mathrm{t} 3$ adipocytes. The results suggest that the CLA-t10c12 caused extra lipid hydroperoxide damage compared to CLA-c9t11, which activated phase 2 detoxification genes. If the cells were exposed for $72 \mathrm{~h}$, apoptosis genes were activated in CLA-t10c12, which mirrored the 50uM $(\sim 164 \mathrm{ng} / \mathrm{ml})$ DHA treatments. Some of the toxicity of DHA could be alleviated by the addition of 100uM alpha-tocopherol (vitamin E) but not enough, as the cells still expressed apoptosis markers at a reduced level after 72h. Changes in lipid metabolism and lipid oxidation and were all observed in the CLA-c9t11 and CLA-t10c12 at $100 \mathrm{uM}(\sim 280 \mathrm{ng} / \mathrm{ml})$ but only the CLAt10c12 was consistent for expression of apoptosis biomarkers after $72 \mathrm{~h}$.

CLA-c9t11 occurs much more commonly than CLAt10c12 in natural system. In typical beef fat, the concentration of CLA-t $10 \mathrm{c} 12$ is between $0.05 \%$ to $0.12 \%$ of total fat, whereas the more common CLAc9t11 is typically around $0.10 \%$ to $0.5 \%$ of total fat [26]. In the milk, the CLA-t10c12 level is usually around $20 \mathrm{mg} / 100 \mathrm{~g}$ of milk fat [1] but in extreme cases, CLA-t10c12 concentrations can reach $60 \mathrm{mg} / 100 \mathrm{~g}$ of milk fat, which is caused by a high concentrate/low forage diet leading to milk fat depression [3]. In normal milk the concentration of CLA-c9c11 is usually $400 \mathrm{mg}$ to $1070 \mathrm{mg} / 100 \mathrm{~g}$ of milk fat. Even if the two isoform of CLA are supplied in equal concentrations, the CLA-c9t11 is preferred. Humans supplemented with $500 \mathrm{mg}$ per day of equal amounts CLA-c9t11 and CLA-t10c12 for 6 months, had blood CLAc9t11 levels reaching approximately $0.2 \%$ of plasma fat (equivalent to a maximum of $\sim 6.5 \mathrm{ug} / \mathrm{ml}$ of plasma) but CLA-t10c12 only reached $0.03 \%$. $(\sim 0.1 \mathrm{ug} / \mathrm{mL}$ of plasma $\sim 0.36 \mathrm{uM}$ ) [27].

Lipid hydroperoxides are generated when the double bonds in PUFAs react to oxidation, mainly through reactive oxidation species (ROS) but also by enzyme processes such as $\mathrm{P} 450$ mediated xenobiotic metabolism. In systems that are experiencing a lot of oxidation or the concentration of PUFA are too high, cellular activity suffers and the newly generated lipid peroxides will react with other targets. The addition of $50 \mathrm{uM} \mathrm{DHA}$ to cells generated lipid peroxide levels of $8.4+/-1.7$ unit in the cells after $72 \mathrm{~h}$ exposure. This was expected since there are 6 double bonds in DHA (all-cis-docosa$4,7,10,13,16,19$-hexaenoic acid). CLA-t10c12 was the next highest producer of lipid peroxides at $7.1+/-0.11$ after 72h, followed closely by the C18:3 and then by the CLAc9t11 at $6.7+/-2.22$ and $6.0+/-0.3$ after 72 h. CLA-t10c12 has only 2 double bonds but there are other targets such as mitochondrial disruption, DNA adducts and oxidized protein that can act as ROS and these may have contributed to the overall oxidative effect [28]. The effect is pronounced however, as shown by the MTT assays of the mature adipocytes which show the loss of cytosol NAD(P)H-dependent oxidoreductase enzymes activity. MTT results generally correlated with the lipid hydroperoxide assay results, for the selected fatty acid treatments. The DHA at 50uM showed the most inhibition, followed by the CLA-t10c12, the C18:3 and then the CLAc9t11. The DHA mixed with $100 \mathrm{uM}$ alpha-tocopherol was also inhibited relative to the C18:2, which suggest that the cell activity was damaged beyond the ROS interaction with lipids or that a higher concentration of alphatocopherol was needed [16]. Brefeldin, a lactone antibiotic which interrupts protein transport, has been shown to block inflammation in 3t3 cultures caused by 50uM CLA-ct10c12 [8] and pre-treatment with $300 \mathrm{uM}$ oleic acid (C18:1) has also been shown to partially prevent CLA-t10c12 induced cellular inflammation [11]. It would be interesting to see if Brefeldin and oleic acid could have a similar effect on DHA treatments, although in cattle fed vitamin $\mathrm{E}$ with trans fatty acids, it has been difficult to see an effect [29].

The oxidative stress the mature adipocytes were experiencing was also indicated by the expression of chemokines in the cell media. Relative to the control treatment of $100 \mathrm{uM} \mathrm{C18;} 2$, the C18:3(n-3) appeared to increase the expression of CCL-11, CCL-5 CxCl-1, VEGF, MCP-1 and adipoQ release into the growth media. DHA and the CLA-t10c12 both increased the CCL11 and CxCl1 signal after $48 \mathrm{~h}$ but only the DHA additionally increased the MCP-1, VEGF and AdipoQ signals. The unique stress of CLA-t10c12 treatment to activate CCL11 and CxCl-1 expression will have to be investigated further; it could be that the 50uM DHA treatment was already too toxic for the cells.

Investigation of cellular apoptosis markers: caspase 3 , BAX and Bcl2 showed a similar effect of stress in the DHA, CLA-t10c12 and C18:3(n-3) treated cells. The expression was significantly stimulated for the caspase 3 gene after 48h, and $72 \mathrm{~h}$ treatment with DHA, CLA-t10c12 and C18:3, which also coincided with the lipid hydroperoxide production and the MTT assay for cell viability. Bcl2 and BAX expression is reported as a ratio with BAX expression being more indicative of apoptosis and $\mathrm{Bcl} 2$ indicative of signalling traffic [30]. BAX was up-regulated in the DHA and CLA-t10c12 treatments after $48 \mathrm{~h}$ but were generally 


\section{Open Access Journal of Agricultural Research}

reduced by $72 \mathrm{~h}$. Bcl 2 was also upregulated in the DHA and C18:3(n-3) treatments after $48 \mathrm{~h}$ but after $72 \mathrm{~h}$ only the DHA treated cells still showed significant stimulation. The relationship between ROS and apoptosis is commonly found among many system studying the effect of novel drugs including cholesterol statins and omega-3 fatty acids [13]. In these trials, these fatty acids also showed a significant effect on expression but the amount of expression was quite high in variability, even after 4 separate trials.

Genes which are activated by ROS and other cellular stresses were modified depending on the fatty acid treatment. The CLA-t10c12, CLA-c9t12 and DHA treatment significantly induced the ark1c1 transcript after $72 \mathrm{~h}$.The aldo-keto reductases (ark1c1) are a group of enzymes which oxidize/reduce aldehydes or ketones to their secondary alcohols [31]. AKR1C1 is involved in the conversion and detoxification of 4-hydroxy-2-nonenol (HNE), which are the oxidation products of fatty acids [32]. The t10c12 and the omega-3 fatty acids C18:3 and DHA significantly induced the MCP-1 (monocyte chemoattractin protein $1 \sim$ CCL2) which is involved in signalling the macrophages along with CCL5. CCL5 (chemoattractin 5, RANTES) is also a macrophage attractant and has been shown to be stimulated by CLA10c12 [25] and we also noted stimulation in the microarray panel and in the cultures treated with C18:3. SOD3 was up regulated but not significantly by t10c12 treatment and was increased in the C18:3(n-3) and DHA treated cells. From the lipid oxidative microarray panel only SOD3 was confirmed to be stimulated by t10c12 over the c9t11, the other oxidation marker targets Duox1, GPX and cog did not show significant stimulation by the treatments by qPCR but these factors warrant further investigation.

Genes involved primarily with lipid metabolism and expression of FABP4 and adipoQ, showed similar effects of significant stimulation by C18:3(n-3) and DHA [33] but only FASN and ACOX1 were significant up regulated by CLA-c9t11 and CLA-t10c12 treatment after 72h. CLAt10c12 did not stimulate adipoQ expression. It is unknown how these same genes respond to apoptosis factors but the evidence suggested that although C18:3 and DHA may be inducing an oxidative stress on the cells; the stress caused by t10c12 is more than just unsaturated bonds and maybe linked to adipoQ expression in CLAc9t11 but not CLA-t10c12 [34]. It might be unique to the position of trans bond at position 10 in CLA-t10c12. In contrast, the trans 9 bond in monounsaturated fat C18:1trans 9, has no effect on FASN and delta-9 desaturation expression $[35,36]$. CLA-t10c12 has been noted as causing an increase in oxidative stress in the mitochondria and this coincides with a disruption in mitochondrial transport and increased carnitine palmitoyl transferase (CPT-1) expression [10]. No significant change was observe in CPT1 expression in this trial but the apoptosis markers BAX and Bcl-2 are often associated with changes in the mitochondrial environment and reflect the oxidative stress.

\section{Conclusion}

The unique mechanism induced by CLA-t10c12 as compared to CLA-C9t11 appears to be through promotion of excessive lipid oxidation and ROS which leads to a variety of down-stream cellular processes. Evidence of this increase in ROS is shown by the increased lipid hydroperoxides, which were also seen in the omega-3 fatty acids treatments of C18:3(n-3) and DHA. However, there are some differences in the cellular response to CLA, the foremost being the lack of adipoQ expression with CLA-t10c12 and an increase in FAS and ACOx1 expression. This difference could be due to the trans-10 bond in the CLA-t10c12, causing it to be poorly absorbed and metabolized in eukaryotic systems [37]. This provides additional warning to food processors on the incorporation of extra trans-fat in the preparation of milk, butter [38] and meat products.

\section{Authors Contribution and Acknowledgment}

Dr Meadus designed, generated and interpreted the data presented in the manuscript. Dr Vahmani assisted in the design of experiments and in the qPCR data. Pascale Duff was the main technician responsible for the molecular data. Dr Zantinge assisted in the molecular data generation and sequencing confirmation on the qPCR products. Dr Turner assisted in the preparation of 3T3 cells and the initial preparation of fatty acids. Dr Dugan is the principal investigator for lipid research at Agri-Food \& Agriculture Canada in Lacombe.

\section{Funding}

This research was supported by Agri-Food and Agriculture Canada under grant \# J-00248.

\section{Conflict of Interest}

The authors declare that the research was conducted in the absence of any commercial or financial relationships that could be construed as a potential conflict of interest. 


\section{Open Access Journal of Agricultural Research}

\section{References}

1. Butler G, Collomb M, Rehberger B, Sanderson R, Eyre $\mathrm{M}$, et al. (2009) Conjugated linoleic acid isomer concentrations in milk from high- and low-input management dairy systems. Journal of the Science of Food and Agriculture 89(4): 697-705.

2. Kramer JKG, Fellner V, Dugan MER, Sauer FD, Mossoba MM, et al. (1997) Evaluating acid and base catalysts in the methylation of milk and rumen fatty acids. Lipids 32(11): 1219-1228.

3. Peterson DG, Matitashvili EA, Bauman DE (2003) Diet-induced milk fat depression in dairy cows results in increased trans-10, cis-12 CLA in milk fat and coordinate suppression of mRNA abundance for mammary enzymes involved in milk fat synthesis. J Nutr 133(10): 3098-3102.

4. Mitchell PL, Karakach TK, Currie DL, McLeod RS (2012) t-10, c-12 CLA dietary supplementation inhibits atherosclerotic lesion development despite adverse cardiovascular and hepatic metabolic marker profiles. PLoS One 7(12): e52634.

5. Dugan MER, Aalhus JL, Schaefer AL, Kramer JKG (1997) The effect of conjugated linoleic acid on fat to lean repartitioning and feed conversion in pigs. Canadian Journal of Animal Science 77(4): 723-725.

6. Qi R, Yang F, Huang J, Peng H, Liu Y, et al. (2014) Supplementation with conjugated linoeic acid decreases pig back fat deposition by inducing adipocyte apoptosis. BMC Vet Res 10: 141.

7. Tsuzuki T, Igarashi M, Iwata $\mathrm{T}$, Yamauchi-Sato $\mathrm{Y}$, Yamamoto T, et al. (2004) Oxidation rate of conjugated linoleic acid and conjugated linolenic acid is slowed by triacylglycerol esterification and alphatocopherol. Lipids 39(5): 475-480.

8. Martinez K, Kennedy A, West T, Milatovic D, Aschner $\mathrm{M}$, et al. (2010) trans-10, cis-12-Conjugated linoleic acid instigates inflammation in human adipocytes compared with preadipocytes. J Bio Chem 285(23): 17701-17712.

9. Reynolds CM, Roche HM (2010) Conjugated linoleic acid and inflammatory cell signalling. Prostaglandins Leukot Essent Fatty Acids 82(4-6): 199-204.

10. Rasooly R, Kelley DS, Greg J, Mackey BE (2007) Dietary trans 10, cis 12-conjugated linoleic acid reduces the expression of fatty acid oxidation and drug detoxification enzymes in mouse liver. Br J Nutr 97(1): 58-66.

11. Reardon M, Gobern S, Martinez K, Shen W, Reid T, et al. (2012) Oleic acid attenuates trans-10, cis-12 conjugated linoleic acid-mediated inflammatory gene expression in human adipocytes. Lipids 47(11): 10431051.

12. Lamkanfi M, Dixit VM (2014) Mechanisms and functions of inflammasomes. Cell 157(5): 1013-1022.

13. Yamasaki M, Miyamoto $Y$, Chujo H, Nishiyama K, Tachibana H, et al. (2005a) Trans10, cis12-conjugated linoleic acid induces mitochondria-related apoptosis and lysosomal destabilization in rat hepatoma cells. Biochim Biophys Acta 1735(3): 176-184.

14. Ahmed AA, Balogun KA, Bykova NV, Cheema SK (2014) Novel regulatory roles of omega-3 fatty acids in metabolic pathways: a proteomics approach. Nutr Metab 11(1): 6 .

15. Kim HK, Della-Fera M, Lin J, Baile CA (2006) Docosahexaenoic acid inhibits adipocyte differentiation and induces apoptosis in 3T3-L1 preadipocytes. J Nutr 136(12): 2965-2969.

16. Yamasaki M, Nishida E, Nou S, Tachibana H, Yamada K (2005b) Cytotoxity of the trans10, cis12 isomer of conjugated linoleic acid on rat hepatoma and its modulation by other fatty acids, tocopherol, and tocotrienol. In Vitro Cell Dev Biol Anim 41(7): 239244.

17. Denizot F, Lang R (1986) Rapid colorimetric assay for cell growth and survival. Modifications to the tetrazolium dye procedure giving improved sensitivity and reliability. J Immunol Methods 89(2): 271-277.

18. van Meerloo J, Kaspers GJ, Cloos J (2011) Cell sensitivity assays: the MTT assay. Methods Mol Biol 731: 237-245.

19. Gill CO, Saucier L, Meadus WJ (2011) Mycobacterium avium subsp. paratuberculosis in dairy products, meat, and drinking water. J Food Prot 74(3): 480-499.

20. Schmittgen TD, Livak KJ (2008) Analyzing real-time PCR data by the comparative C(T) method. Nat Protoc 3(6): 1101-1108. 


\section{Open Access Journal of Agricultural Research}

21. Bustin SA, Benes V, Garson JA, Hellemans J, Huggett J, et al. (2009) The MIQE guidelines: minimum information for publication of quantitative real-time PCR experiments. Clin Chem 55(4): 611-622.

22. Yuan JS, Reed A, Chen F, Stewart CN (2006) Statistical analysis of real-time PCR data. BMC Bioinformatics 7: 85.

23. Shaw B, Lambert S, Wong MH, Ralston JC, Stryjecki C, et al. (2013) Individual saturated and monounsaturated fatty acids trigger distinct transcriptional networks in differentiated 3T3-L1 preadipocytes. J Nutrigenet Nutrigenomics 6(1): 1-15.

24. Prostek A, Gajewska M, Kamola D, Balasinska B (2014) The influence of EPA and DHA on markers of inflammation in 3T3-L1 cells at different stages of cellular maturation. Lipids Health Dis 13: 3.

25. Poirier H, Shapiro JS, Kim RJ, Lazar MA (2006) Nutritional supplementation with trans-10, cis-12conjugated linoleic acid induces inflammation of white adipose tissue. Diabetes 55(6): 1634-1641.

26. Rule DC, Broughton KS, Shellito SM, Maiorano G (2002) Comparison of muscle fatty acid profiles and cholesterol concentrations of bison, beef cattle, elk, and chicken. J Anim Sci 80(5): 1202-1211.

27. Zlatanos SN, Laskaridis K, Sagredos A (2008) Conjugated linoleic acid content of human plasma. Lipids Health Dis 7: 34.

28. Stachowska E, Baskiewicz-Masiuk M, Dziedziejko V, Gutowska I, Baranowska-Bosiacka I, et al. (2008) Conjugated linoleic acid increases intracellular ROS synthesis and oxygenation of arachidonic acid in macrophages. Nutrition 24(2): 187-199.

29. Mapiye C, Dugan ME, Juarez M, Basarab JA, Baron VS (2012) Influence of alpha-tocopherol supplementation on trans-18:1 and conjugated linoleic acid profiles in beef from steers fed a barleybased diet. Animal 6(11): 1888-1896.

30. Ip C, Ip MM, Loftus T, Shoemaker S, Shea-Eaton W (2000) Induction of apoptosis by conjugated linoleic acid in cultured mammary tumor cells and premalignant lesions of the rat mammary gland. Cancer Epidemiol Biomarkers Prev 9(7): 689-696.
31. Petrash JM (2004) All in the family: aldose reductase and closely related aldo-keto reductases. Cell Mol Life Sci 61(7-8): 737-749.

32. Ayala A, Munoz MF, Arguelles S (2014) Lipid peroxidation: production, metabolism, and signaling mechanisms of malondialdehyde and 4-hydroxy-2nonenal. Oxid Med Cell Longev 2014: 360438.

33. Duda MK, O'Shea KM, Tintinu A, Xu W, Khairallah RJ, et al. (2009) Fish oil, but not flaxseed oil, decreases inflammation and prevents pressure overloadinduced cardiac dysfunction. Cardiovasc Res 81(2): 319-327.

34. Minemoto $Y$, Adachi S, Shimada Y, Nagao T, Iwata T, et al. (2003) Oxidation kinetics for cis-9,trans-11 and trans-10,cis-12 isomers of CLA. Journal of the American Oil Chemists' Society 80(7): 675-678.

35. Vahmani P, Meadus WJ, da Silva ML P, Mapiye C, et al. (2015a) Effects of beef trans-18:1 from conventional versus flax/hay fed cattle on global gene expression in HepG2 liver cells. Journal of Glycomics Lipidomics 5(4): 66.

36. Vahmani P, Meadus WJ, Mapiye C, Duff P, Rolland DC, et al. (2015b) Double Bond Position Plays an Important Role in Delta-9 Desaturation and Lipogenic Properties of Trans 18:1 Isomers in Mouse Adipocytes. Lipids 50(12): 1253-1258.

37. Kuhnt K, Baehr M, Rohrer C, Jahreis G (2011) Trans fatty acid isomers and the trans-9/trans-11 index in fat containing foods. Eur J Lipid Sci Technol 113(10): 1281-1292.

38. Roy A, Chardigny JM, Bauchart D, Ferlay A, Lorenz S, et al. (2007) Butters rich either in trans-10-C18:1 or in trans-11-C18:1 plus cis-9, trans-11 CLA differentially affect plasma lipids and aortic fatty streak in experimental atherosclerosis in rabbits. Animal 1(3): 467-476. 